\title{
Role of Veertarvadi Gana in Mutrashmari : A Review Study
}

\section{B. N. Gadve ${ }^{1}$, Sheetal M. Balkunde ${ }^{* 2}$}

1. Associate professor and Guide, 2. P.G. Scholar,

\author{
Department of Shalyatantra,
}

C.S.M.S.S. Ayurveda Mahavidyalaya, Kanchanwadi, Aurangabad.

*Corresponding Author: Email: drsheetalbalkunde@gmail.com

\section{Abstract:}

Group of drug described as Gana in Samhita. Veertarvadi Gana is one out of 37 Ganas mentioned in Sushruta Samhita Sutrasthan 38/4, with 18 herbs in it. They are indicated in Vaatvikar, Ashmari, Sarkara, Mutrakrichha, Mutraghat. Ashmari is very common problem due to todays lifestyle, Dietary habits, it contributes more in formation of Mutrashmari (Urinary Calculus). Aacharya Sushruta included it in Ashthaumahagada. The main aim of this article is to review the importance \& utility of Veertarvadi Gana in Mutrashmari.

Keywords: Veertarvadi Gana, Mutrashmari, Urinary Calculus.

\section{Introduction:}

Ashmari (Calculi) comprises of two words i.e. 'Ashma' \& 'Ari. Ashma means a Stone \& Ari means Enemy.

Ashmari Specifically called as Mutrashmari (Urolithiasis), is a disease of Mutravahastrotas (Urinary tract) \& involves formation of stone resulting into severe pain as given by Enemy ${ }^{1}$. Ashmari has been mentioned in all our ancient texts but Aacharya Sushruta has described it elaborately \& included it in Ashthaumahagada ${ }^{2}$.

Urinary Calculus is a stone like body composed of Urinary salts bound together by a colloid matrix of organic materials. It consists of a nucleus around which concentric layers of Urinary salts 
are deposited ${ }^{3}$. Urinary calculus below 5 $\mathrm{mm}$ size are flush out automatically with urine, more than $5 \mathrm{~mm}$ size causes pain, dysuria, haematuria ${ }^{4}$. According to modern science surgery is the only one treatment \& very less medicines are available but recurrence is common.

In Ayurveda group of drugs are mentioned to treat Mutrashmari among them the Virtarvadi Gana mentioned in Sushruta Samhita Sutrasthan 38/4. This Gana contains 18 plants as Veertaru, Sahachradvya, Darbha, Vrikshadani, Gundra, Nala, Kusha, Kasha, Ashmabheda, Agnimantha, Morata, Vasuka, Vasira, bhalluka, Kurantaka, Indivara, Kapotvanga, Shwadanshtra. They are indicated in Vaatvikar, Ashmari, Sarkara, Mutrakrichha, Mutraghat ${ }^{5}$.

Samprapti (Pathophysiology) $)^{6}$ :

Non adoption of Vamana, Virechanadi Panchakarma, Mithya Aahar-vihar (Unhealthy diet \& life style) due to this Kapha Dosha is vitiated \& combines with urine reaches the Basti (Urinary bladder) \& stay there produces Ashmari .

\section{Prodromal Signs \& Symptoms (Ayurvedic aspects) $^{7}$ :}

Pain around the Umbilicus, Urinary bladder region, penis \& other nearby areas during micturition. Obstructed Urinary flow, scattering of urine, haematuria, colour of urine resembles like Gomedak (Hessonite stone), turbid urine, Sand like particles passing along with urine. Pain aggravated by jumping, swimming, running, riding, walking etc.

Classification of Ashmari ${ }^{8}$ :

1) SHLESHMAASHMARI: Ashmari is white in color, slimyin texture $\&$ as big as a Hens egg or having colour of Madhuka flower.

2) PITTAASHMARI: Ashmari is reddish, yellowish \& resembles the colour of Honey \& seed of Bhallatka.

3) VATAASHMARI: Ashmari is bluish dusty in colour, hard, irregular, rough \& throny like Kadamba Flowers.

4) SHUKRA ASHMARI: Occurs in adults only due to suppression of ejaculation for months or years \& frequent coitus or coitus interruption. The semen to be ejaculated gets obstructed ,condensed \& brought in between the scrotum \& penis by Vata. 
This calculi break at its place when squeezed by hand.

Shleshma Ashmari can be correlated with the phosphatic calculus, Pitt Ashmari with the Uric acid calculus, Vata Ashmari with oxalate stones \& Shukra Ashmari with spermolith or seminal or spermatic concentrations.

Aim: To study the role of Veertarvadi Gana in Mutrashmari.

Method \& Material : Classical texts of Ayurveda, journals, Internet.

Veertarvadi Gana:

1) VEERTARU:

Latin name- Dichrostachys cineria

Family- Leguminosae Local

Name- Vellantar

Gun- Laghu, Ruksha Ras- Tikta, Kashay

Virya- Ushna Vipak-

Katu

Karma \& Doshaghnata - Kapha, Vatashamak, Ashmarinashan, Mutral, Vedanasthapan

Prayojyanga- Mula

Properties - Useful in retention of urine, Calculi ${ }^{9}$.
2) Sahachardavaya-

Latin name- 1) Shwet SahacharBarleria cristata

2) Nil Sahacahar- $B$. wild strigosa

Family- Acantheaceae

Local Name - Sayreyak, Koranti

Gun- Laghu

Ras- Tikta, Madhur

Virya- Ushna

Vipak- Katu

Karma \& Doshaghnata- Kapha, Vataghna, Mutral, Mutrakrucchahar

Prayojyanga- Panchang (Visheshtah Patra) Properties - Diuretic

\section{3) Darbha :}

Latin name- Imparata cylindrica

Family- Gramineae

Local Name- Dabha, Kusha

Gun- Laghu, Snighdha

Ras- Madhur, Kashay

Virya- Shit

Vipak- Madhur

Karma \& Doshaghnata- Tridoshaghna, Ashmarinashak, Mutral, Dahaprashaman Prayojyanga- Mul 
Properties - Diuretic, useful in urinary disorder ${ }^{10}$.

\section{4) Vrukshadani:}

\section{Latin name- Dendrophthoe falcata}

Family- Loranthaceae

Local Name- Bandak, Bandgul

Gun- Laghu, Ruksha

Ras- Kashay, Tikta, Madhur

Virya- Shit

Vipak- Katu

$\begin{array}{lr}\text { Karma } \quad \& \quad & \text { Doshaghnata- } \\ \text { Ashmarinashak, } & \text { Mutrajanan, } \\ \text { Mutrakrucchahar } & \end{array}$

Prayojyanga- Panchang

Properties - Diuretic ${ }^{11}$.

\section{5) Gundra :}

Latin name- Typha elephantina Roxb.

Local Name - Pater, Godar

Family- Typhaceae

Gun- Shit

Ras- Kashay, Madhur

Virya- Shit

Vipak- Madhur
Karma \& Doshaghnata- Mutral,

Dahanashak, Ashmarinashak

Prayojyanga- Mul

Properties - Diuretic ${ }^{12}$

6) Nala:

Latin name- Arundo donox

Family- Gramineae

Local Name- Nala, Narasal

Gun- Laghu, Snigdha

Ras- Madhur, Kashay, Tikta

Virya- Shit

Vipak- Madhur

Karma \& Doshaghnata- Mutral, Dahashamak, Bastishothahar,

Mutakrucchahar

Prayojyanga- Mul

Properties - Diuretic ${ }^{13}$, lithotripsic, used in cystitis and Dysuria ${ }^{14}$.

\section{7) Kusha:}

Latin name- Desmotachya bipinnata

Family- Gramineae

Local name- Kusha

Gun- Laghu, Snigdh

Ras- Madhur, Kashay 
Virya- Shit

Vipak- Madhur

Karma \& Doshaghnata- Asmarinashak,

Mutral, Bastishulnashak

Prayojyanga- Mul

Properties - Diuretic, Lithotripsic ${ }^{15}$.

8) Kasha :

Latin name- Saccharum spontaneum

Family- Gramineae Local

Name- Kasaie

Gun- Laghu, Snighdha Ras-

Madhur, Kashay

Virya- Shit

Vipak-

Madhur

Karma \& Doshaghnata-

Vatapittashamak, Mutravirechaniya,

Ashmaribhedan

Prayojyanga- Mula

Properties

- Diuretic, lithotripsic ${ }^{16}$.

9) Ashmabheda:

Latin name- Bergenia Ligulata

Family- Saxifragaceae

Local Name- Pashanbhed

Gun- Laghu, Snigdha, Tikshna Ras-

Kashay, Tikta
Virya- Shit

Vipak- Katu

Prabhav - Ashmaribhedan

Karma \& Doshaghnata-

Tridoshshamak, Ashmaribhedan, Mutral

Prayojyanga- Mul

Properties - Lithotripsic, used in dysuria $^{17}$

10) Agnimanth:

Latin name- Premna intergrifolia

Family- Verbenaceae

Gun- Ruksha, Laghu

Ras-

Tikta, Katu, Kashay, Madhur

Virya- Ushna

Vipak-

Katu

Karma \& Doshaghnata-

Kaphavatashamak, Shothahar,

Vedanasthapan

Prayojyanga- Mul, Patra

Properties - Analgesic ${ }^{18}$.

11) Morata:

Latin name- Marsdenia tenacissima

Family- Asclpiadaceae

Local name - Murva

Gun- Guru, Ruksha 
Ras- Tikta, Kashay

Virya- Ushna

Vipak- Katu

Karma \& Doshaghnata- Tridoshnashak

Prayojyanga- Mul

Properties - Antispasmodic, used for colic pain ${ }^{19}$.

12) Vasuka:

Latin name- Osmanthns fragrans

Family-Sapotaceae

Local name - Bruhat bakkul

Gun- Guru

Ras- Kashay, Katu

Virya- Shit

Vipak- Katu

Karma \& Doshaghnata- Pittakaphashamak, Bastishothahar

Prayojyanga- Twak, Pushpa, Phala Properties - Used in Cystitis ${ }^{20}$.

13) Vasira:

Latin name- Achyranths aspera

Family- Amaranthaceae

Local name- Apamarg
Gun- Laghu, Ruksha, Tikshna RasKatu, Tikta

Virya- Ushna

Vipak- Katu

Karma \& Doshaghnata- Kaphavatashamak, Mutral, Ashmarinashan

Prayojyanga- Mul, Tandul, Pancahng Properties - Analgesic, Diuretic ${ }^{21}$.

14) Bhalluka:

Latin name- Oroxynum indicum

Family- Bignonaiaceae

Local name- Shonyak

Gun-Laghu, Ruksha

Ras- Kashay, Madhur, Tikta

Virya- Ushna

Vipak- Katu

Karma \& DoshaghnataKaphavatshamak, Mutral, Bastishothahar Prayojyanga- Mul,Twak

Properties - Diuretic ${ }^{22}$.

15) Kurantak:

Latin name- Barleria prionitis

Family-Acanthaceae

Local name- Pit Sayreyak 
Gun- Laghu

Ras- Tikta, Madhur

Virya- Ushna

Vipak- Katu

Karma

\&

Doshaghnata-

Kaphavatshamak, Mutral,

Mutrakrucchahar

Prayojyanga- Panchang (Visheshtaha Patra) Properties - Diuretic ${ }^{23}$.

16) Indivara:

Latin name- Nelumbo nucifera

Family- Nymphaeaceae

Local name- Nilkamal

Gun- Laghu, Snigdha, Picchil

Ras- Kashay, Madhur, Tikta

Virya- Shit

Vipak- Madhur

Karma \& Doshaghnata-

Kaphapittashamak, Mutrakrucchahar, Mutravirechaniya

Prayojyanga- Panchang (Visheshtah Pushpa, Beej, Mul)

Properties - Diuretic ${ }^{24}$.

\section{7) Kapotvanga:}

Latin name- Herpestris moniera
Family- Scrophulariaceae

Local Name - Brahmi

Gun- Laghu

Ras- Kashay, Tikta

Virya- Shit

Vipak- Madhur

Karma \& Doshaghnata- Tridoshnashak, Mutral

Prayojyanga- Panchang

$$
\text { Properties - Diuretic }{ }^{25} \text {. }
$$

18) Shwadanshtra:

Latin name- Tribulus terresteris

Family-Zygophyllaceae

Local name- Gokshur

Gun- Guru, Snigdha

Ras- Madhur

Virya- Shit

Vipak- Madhur

Karma \& DoshaghnataVatpittashamak, Ashmarinashan, Mutral

Prayojyanga- Phal, Mul

Properties- Diuretic, Lithontriptic ${ }^{26}$.

Discussion:

On reviewing the all ingredients in this Veertarvadi Gana Darbha, Kusha, 
Gundra, Morata (Murva) are Sandigdha Dravyas (controversial drug).

Controversial is a term used for medicinal plants having various sources.

There is enormous controversy between Kusha and Darbha in Ayurveda literature.

Though the synonyms actions and indications of Kusha, Darbha are mentioned together in different texts, The most of the Nighantus accepted both Kusha and Darbha as two different varieties and mentioned as synonyms for each other ${ }^{27}$.

Typha elephantina $\operatorname{roxb}^{28}$, Cyperus rotundus $^{29}$, Callicarpa macrophylla ${ }^{30}$ are considered as Gundra in different books.

Marsdenia tenacissima ${ }^{31}$, Clematis gouriana $^{32}$, Sansevieria roxburghiana ${ }^{33}$, Chonemorpha fragrans ${ }^{34}$ are considered as Murva in different books.

Conclusion :

Maximum Herbs are Ashmaribhedak (Lithotripsic), Mutral (Diuretic), Mutrakrucchahar and useful in diseases of Bladder and Kidney.

\section{References:}

1. Monika et al. conceptual review on etiopathogenesis and management of urolithiasis in Ayurveda with proven clinical trial, IJHSR, vol 6, Issue:10, oct 2016.

2. Sushrut, Shastri Ambika Dutta, Sushrut Samhita, Purvardha, chaukhamba publication ;2014, Sutra Sthan 33/4-5, page no.163.

3. DAS S., A concise textbook of surgery $3^{\text {rd }}$ edition, chapter 57 , page no. 1165 .

4. Anju M.Hadke, Role of Ayurveda in Mutrashmari (urolithiasis) a review, Ayurlog, Vol $6^{\text {th }}$, Issue: $5^{\text {th }}$ , august 2018.

5. Sushrut, Shastri Ambika Dutta, Sushrut Samhita, Purvardha, chaukhamba publication ;2014, Sutra Sthan 38/11, page no.183.

6. Sushrut, Shastri Ambika Dutta, Sushrut Samhita, Purvardha, chaukhamba publication ;2014, Nidan Sthan 3/4, page no.311.

7. Sushrut, Shastri Ambika Dutta, Sushrut Samhita, Purvardha, chaukhamba publication ;2014, Nidan Sthan 3/7, page no.312.

8. Sushrut, Shastri Ambika Dutta, Sushrut Samhita, Purvardha, chaukhamba publication ;2014, Nidan Sthan 3/8-9-10-12, page no. 312,313 .

9. Vd. Gogte V.M., Ayurvedic pharmacology \& Therapeutic uses 
of medicinal plants (Dravyagunavigyan), English edition, oct 2000 , page no.723

10. A.Rama Murthy ${ }^{1}$,Medicinal importance of Darbha-A review, J Ayu Herb med. ,Vol 2,Issue 3,may-june 2016.

11. Vd. Gogte V.M., Ayurvedic pharmacology \& Therapeutic uses of medicinal plants (Dravyagunavigyan), English edition, oct 2000, page no.677.

12. Rahman et al, Evaluation of analgesic activity of the different fractions of Typha elephantina roxb., IJP (2014), Vol 1, Issue 6: 380-383.

13. Ali Esmail Al-snafi,The constituents and biological effects of Arundo donax-A review, Vol 6, Issue 1, IJPR,2015,34-40.

14. Vd. Gogte V.M., Ayurvedic pharmacology \& Therapeutic uses of medicinal plants (Dravyagunavigyan), English edition, oct 2000, page no.644.

15. Vd. Gogte V.M., Ayurvedic pharmacology \& Therapeutic uses of medicinal plants (Dravyagunavigyan), English edition, oct 2000, page no.589.

16. Database on medicinal plants used in Ayurveda,CCRAS, Vol 8,page no.201.
17. Vd. Gogte V.M., Ayurvedic pharmacology \& Therapeutic uses of medicinal plants (Dravyagunavigyan), English edition, oct 2000, page no.663.

18. Vd. Gogte V.M., Ayurvedic pharmacology \& Therapeutic uses of medicinal plants (Dravyagunavigyan), English edition, oct 2000, page no.287.

19. Database on medicinal plants used in Ayurveda,CCRAS, Vol 8, page no.272.

20. Vd. Gogte V.M., Ayurvedic pharmacology \& Therapeutic uses of medicinal plants (Dravyagunavigyan), English edition, oct 2000, page no.675.

21. Vd. Gogte V.M., Ayurvedic pharmacology \& Therapeutic uses of medicinal plants (Dravyagunavigyan), English edition, oct 2000, page no.295.

22. Database on medicinal plants used in Ayurveda,CCRAS, Vol 2, page no.493.

23. D.Banerjee, Bareleria prionitis Linn:A Reviewvof its traditional uses, Phytochemistry, Pharmacology and toxicity, RJphyto, year 2012, Vol 6, Issue 2, page no.31-41

24. Vd. Gogte V.M., Ayurvedic pharmacology \& Therapeutic uses 
of medicinal plants (Dravyagunavigyan), English edition, oct 2000, page no.583.

25. Vd. Gogte V.M., Ayurvedic pharmacology \& Therapeutic uses of medicinal plants (Dravyagunavigyan), English edition, oct 2000, page no.438.

26. Database on medicinal plants used in Ayurveda,CCRAS, Vol 3, page no.229.

27. A.Rama Murthy ${ }^{1}$, Medicinal importance of Darbha-A review, J Ayu Herb med. ,Vol 2, Issue 3, may-june 2016.

28. Pro.Chunekar krushnchandra, Bhavaprakash Nighantu, Chaukhambha Bharati Academy, Varanasi, page no 381.

29. Vd.

Gogte

V.M.,

Dravyagunavigyan, vaidyamitra prakashan, 2008, page no.576.
30. Vd.

Gogte

V.M.,

Dravyagunavigyan, vaidyamitra prakashan, 2008, page no.509.

31. Sharma priyavat, Darvyaguna vidnyan, chaukhamba bharti academy, Varanasi, part 2, page no.699.

32. Vd.

Gogte V.M., Dravyagunavigyan, vaidyamitra prakashan, 2008, page no.580581.

33. Sharma priyavat, Darvyaguna vidnyan, chaukhamba bharti academy, Varanasi, part 2, page no.769.

34. Varsha Tiwari et al, Phytopharmacological Overview on Controversial Drug: Murva, Traditonal and Folk Herbal Medicine:Recent Researches vol.2.

\section{Cite article:}

Role of Veertarvadi Gana in Mutrashmari: A Review Study

B. N. Gadve, Sheetal M. Balkunde

Ayurlog: National Journal of Research in Ayurved Science- 2019; (7)(3): 1- 10 